Service social

\title{
Le placement des enfants au Québec, des années 1930 à aujourd'hui. Une mesure trop souvent utilisée? Constatations et hypothèses
}

\section{Renée Joyal et Carole Chatillon}

Volume 45, numéro 2, 1996

Droit et pratiques sociales

URI : https://id.erudit.org/iderudit/706725ar

DOI : https://doi.org/10.7202/706725ar

Aller au sommaire du numéro

Éditeur(s)

École de service social de l'Université Laval

ISSN

1708-1734 (numérique)

Découvrir la revue

Citer cet article

Joyal, R. \& Chatillon, C. (1996). Le placement des enfants au Québec, des années 1930 à aujourd'hui. Une mesure trop souvent utilisée? Constatations et hypothèses. Service social, 45(2), 31-50. https://doi.org/10.7202/706725ar
Résumé de l'article

Depuis plusieurs décennies, nombre d'études et de rapports aussi bien privés que publics posent le problème du placement des enfants au Québec et formulent l'hypothèse du recours excessif à cette mesure. On aurait trop souvent retiré des enfants de leur milieu familial pour les placer en institution ou en famille d'accueil, alors que, dans bien des cas, des mesures de soutien à domicile auraient pu préserver la cohésion familiale. Le présent texte retrace l'évolution du placement au Québec depuis les années 1930, tant du point de vue qualitatif que quantitatif, et propose quelques éléments juridiques et socioculturels explication de la situation particulière du Québec dans ce domaine. 


\section{Le placement des enfants au Québec, des années 1930 à aujourd'hui. Une mesure trop souvent utilisée? Constatations et hypothèses}

Renée JOYAL

Avocate et professeure Département des sciences juridiques Université du Québec à Montréal

Carole CHATILLON Avocate et agente de recherche Université du Québec à Montréal

Au Québec, la question du retrait de l'enfant de son milieu naturel fait l'objet, depuis plusieurs décennies, de questionnements et de débats persistants. Il y aurait chez nous une «tradition» de recours excessif au placement des enfants et une sousutilisation corrélative de mesures efficaces de soutien à domicile de ceux-ci et de leur famille ${ }^{1}$. On aurait tendance à retirer les enfants de leur milieu familial et à les placer en centre ou en famille d'accueil sans que des motifs suffisants le justifient dans tous les cas. 
En 1990, le Conseil des affaires sociales rendait public un Avis relatif à cette question. Selon ce document, le placement en milieu substitut était toujours à cette date le recours le plus fréquemment utilisé pour venir en aide aux enfants se trouvant sous la protection de l'État. Il en allait notamment ainsi pour les enfants dont la situation était prise en charge par les directions de la protection de la jeunesse (DPJ) pour l'année 1989: $55 \%$ d'entre eux vivaient en centre ou en famille d'accueil. Le même Avis affirmait également que le Québec connaissait pour cette même année des taux de placement supérieurs à ceux du reste du Canada, en particulier de l'Ontario. Alors que 8,4/1000 enfants québécois étaient placés en milieu substitut en 1989, seulement 4,4 / 1000 l'auraient été dans la province voisine ${ }^{2}$.

La persistance de cet état de fait est d'autant plus difficile à expliquer que, depuis plusieurs décennies, cette tendance québécoise a été maintes fois remise en cause tant dans le discours public que privé sur la question. On relève les premières traces de cette préoccupation dans le rapport de la Commission des assurances sociales de 1933 (rapport Montpetit) ${ }^{3}$. En 1995, plus de soixante ans plus tard, le ministère de la Santé et des Services sociaux rend publics, conjointement avec d'autres organismes gouvernementaux et paragouvernementaux, deux importants documents intitulés Maintenant et pour l'avenir... La jeunesse et Vers un continuum de services intégrés à la jeunesse $e^{4}$, qui énoncent de nombreuses recommandations destinées à limiter le placement des enfants et à favoriser la mise en œuvre de mesures de rechange appropriées. Entre ces deux dates, nombre d'études et de rapports ont abordé cette épineuse question.

À travers tous ces documents et à l'aide de données démographiques, il importe tout d'abord de dresser un portrait de l'évolution du placement des enfants au Québec. Qu'en est-il depuis les années 1930 et 1940, tant du point de vue qualitatif que quantitatif? Si vraiment «retard» il y a, par rapport à d'autres sociétés, quant à la diminution du recours au placement et à la mise en place de mesures alternatives efficaces, il convient d'examiner divers éléments contextuels susceptibles d'expliquer ce phénomène. 


\section{LE PLACEMENT DES ENFANTS AU QUÉBEC DEPUIS CINQUANTE ANS: CHIFFRES ET DISCOURS}

\section{Une évolution statistique étonnante}

Alors qu'on aurait pu croire, de prime abord, à une baisse progressive du taux des enfants placés depuis 1945, en raison, notamment, de la diminution du taux de natalité, de l'amélioration des soins de santé à la population et de l'accroissement de l'aide fournie par l'État-providence jusque dans les années 1970 tout au moins, il n'en est rien dans les faits. La proportion des enfants placés suit une courbe ascendante qui culmine en 1972 et fléchit par la suite jusqu'en 1993. Les données provisoires disponibles pour 1994 laissent voir une stabilisation du taux au-dessous de 7 / 1000. Le tableau et le graphique qui suivent illustrent cette évolution ${ }^{5}$.

Enfants du Québec placés en institution ou en familles d'accueil (ratio / 1000 enfants)

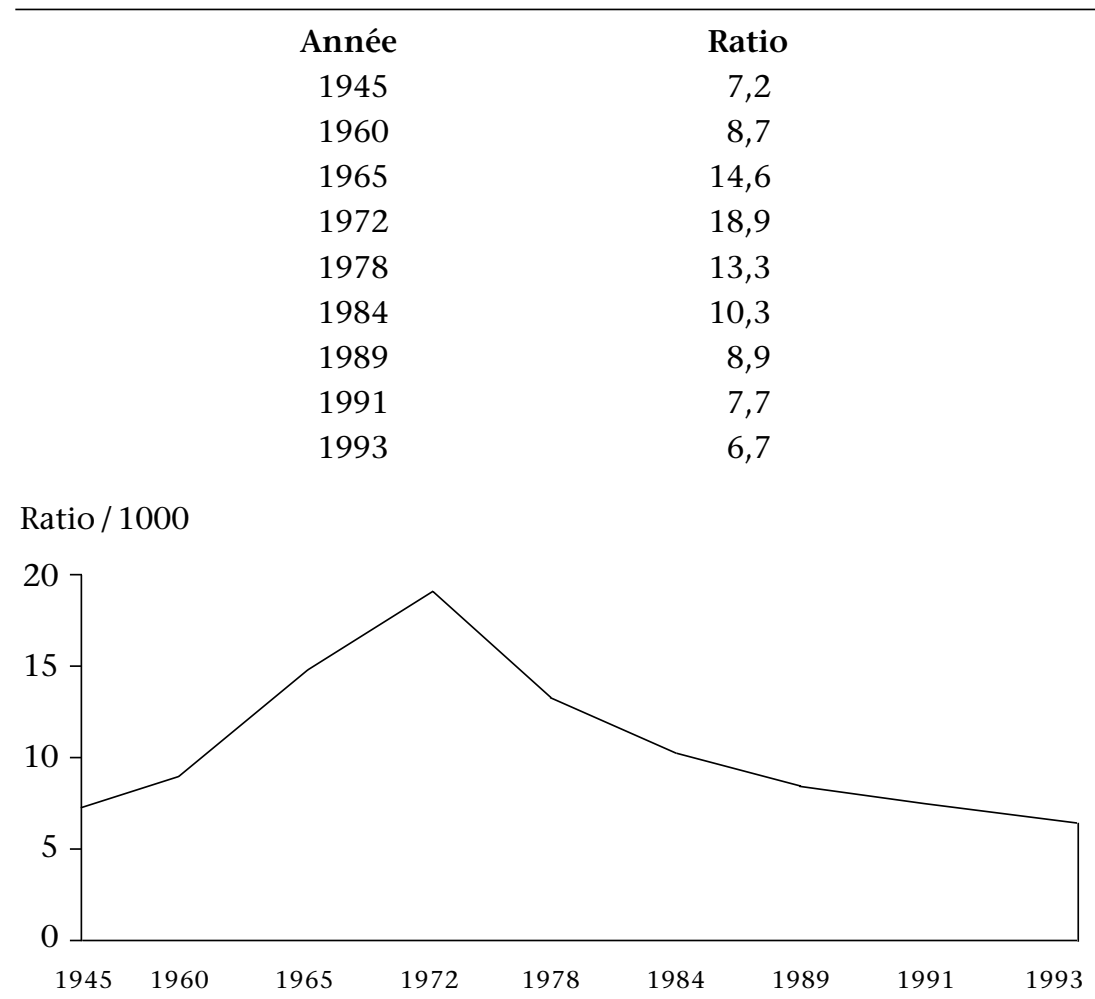




\section{Quelques données qualitatives et quantitatives}

De nombreux documents publics et privés parus entre les années 1930 et aujourd'hui fournissent divers renseignements sur les enfants placés en milieu substitut. Sans apporter d'explications définitives quant aux motifs pour lesquels les placements étaient effectués, ces diverses données jettent un éclairage intéressant sur la situation familiale des enfants placés, tant du point de vue économique que social, ainsi que sur l'évolution des pourcentages de placement.

\section{L'environnement familial}

Le rapport de la Commission des assurances sociales, déposé en 1933, révèle que seulement $12 \%$ des enfants recueillis dans des institutions sont orphelins de père et de mère, alors que $67 \%$ d'entre eux sont orphelins de père ou de mère; il y en a donc $21 \%$ dont les deux parents sont vivants ${ }^{6}$. Au total, $88 \%$ des enfants placés en institution durant les années 1930 ont encore leur père ou leur mère ou leurs deux parents. Même si l'on tient compte des enfants délinquants placés dans des écoles de réforme, qui représentent moins de $4 \%$ des enfants placés, ces données ne peuvent manquer de susciter des interrogations: les enfants concernés étaient-ils tous abandonnés ou avaient-ils été victimes d'abus ou de négligence grave de la part de leurs parents?

Dans une étude publiée en 1930, Arthur Saint-Pierre, traitant de l'assistance institutionnelle dans la province de Québec, faisait déjà remarquer que les institutions de charité y «hospitalisent» trois fois plus d'enfants «indigents» que celles de l'Ontario $^{7}$. Il y a là un indice intéressant de l'ampleur du placement institutionnel des enfants dans le Québec des années 1930.

Plus tard, en 1950, une enquête effectuée auprès de 85 jeunes femmes ayant vécu à l'orphelinat durant au moins cinq ans révèle que seulement $17 \%$ d'entre elles étaient complètement orphelines, alors que $15 \%$ venaient de familles démunies ${ }^{8}$. Une étude analogue menée au même moment dans la région de Québec montre que seulement $12 \%$ des enfants placés l'étaient en raison de leur situation d'orphelins ${ }^{9}$.

Même après 1960, il semble qu'on ait eu recours au placement quand la situation familiale de l'enfant aurait justifié un autre type de soutien. Une étude menée en 1963 par le Conseil des Cuvres de Montréal arrive à la conclusion que des enfants sont placés, alors que leur mère "présente un élément de 
stabilité au foyer». Sur 7000 enfants placés dans la région de Montréal à cette époque, on en dénombre plus de 400 qui le sont en dépit de la stabilité de leur mère ${ }^{10}$.

Autre son de cloche inquiétant, cette fois à l'échelle de la province. Un rapport rendu public en 1979 par le ministère des Affaires sociales et intitulé "Opération 30000 » (du nom du programme dont il découle) apporte certaines précisions sur la situation familiale des enfants placés: «...un cinquième des enfants... sont orphelins des deux parents. Pour près du quart des enfants placés, les praticiens ne connaissent pas l'adresse de l'un des deux parents, pour $15 \%$ ils n'ont aucune information au dossier concernant l'adresse des deux parents et pour $39 \%$, l'adresse des parents est connue». Le même rapport révèle que $48 \%$ des enfants vivant en milieu substitut ont une personne chez qui ils peuvent retourner vivre et que, dans $41 \%$ des cas, celle-ci est le père ou la mère de $l^{\prime} e n f a n t^{11}$. Des écrits subséquents font état de la forte proportion d'enfants placés issus de familles monoparentales ${ }^{12}$.

Toutes ces données, aussi partielles et mouvantes soientelles, se rejoignent néanmoins sur un point. Le retrait de l'enfant de son milieu naturel et le placement de celui-ci en milieu substitut ont longtemps et souvent été faits au Québec sans que cette pratique ait été justifiée par la situation d'abandon de l'enfant ou par des abus graves commis à son égard par ses parents ou ses gardiens. C'est donc ailleurs qu'il faut chercher les motifs qui ont pu être à l'origine de ces placements.

\section{La pauvreté des parents}

Dans de nombreux cas, il semble que ce soit la mauvaise situation économique des parents qui ait conduit au placement des enfants.

Selon une enquête effectuée au cours des années 1940 dans le diocèse de Trois-Rivières, $39 \%$ des enfants placés à l'orphelinat le sont en raison de la pauvreté de leurs parents, alors que seulement $5 \%$ d'entre eux sont orphelins de père et de mère ${ }^{13}$. Des résultats similaires sont obtenus au début des années 1950 pour la région de Québec où $30 \%$ des enfants placés se retrouvent en institution à cause des difficultés économiques de leurs parents ${ }^{14}$. L'étude publiée par le Conseil des Cuvres de Montréal en 1963 conclut pour sa part que bon nombre d'enfants placés pourraient demeurer avec leur mère si celle-ci recevait des allocations sociales raisonnables. Pourtant, plus de quinze ans plus 
tard, le rapport «Opération 30000 » révèle que $80 \%$ des enfants placés en milieu substitut proviennent de familles vivant sous le seuil de la pauvreté ${ }^{15}$. Cette variable n'aurait fait que croître durant les deux dernières décennies ${ }^{16}$.

D'autres facteurs, tels l'état de santé des parents ou de l'un $\mathrm{d}^{\prime} \mathrm{eux}^{17}$ ou encore la séparation des parents ${ }^{18}$, semblent avoir contribué à de nombreux placements.

Le «sommet» de 1972

Les données statistiques relatées sous la rubrique précédente font voir, on s'en souvient, une courbe ascendante qui atteint son sommet en 1972 pour fléchir par la suite. Certaines études ont tenté d'expliquer cette trajectoire étonnante. Alors que la population des enfants de moins de dix-huit ans augmente à peine de 1961 à $1971^{19}$, l'essor remarquable du placement en famille d'accueil fait doubler le nombre d'enfants qui reçoivent des services en dehors de leur milieu pendant la même période ${ }^{20}$. La situation se présente très différemment en Ontario, où le placement en famille d'accueil ne jouit pas d'une telle popularité. Résultat: le Québec place alors $40 \%$ plus d'enfants que la province voisine ${ }^{21}$.

Le recours au placement connaît cependant une baisse significative chez nous durant la période 1973-1978. La diminution s'effectue de façon progressive dans le cas du placement en famille d'accueil, alors que le placement institutionnel fléchit surtout vers la fin de la décennie. Il faut dire que la population des enfants de moins de dix-huit ans diminue d'environ 300000 durant cette période. De plus, "l'abaissement de l'âge de la majorité de vingt et un à dix-huit ans et, dans une certaine mesure, la prise de conscience chez les administrateurs et les praticiens du fait que le Québec avait un nombre d'enfants placés plus élevé qu'ailleurs » peuvent contribuer à expliquer la baisse observée ${ }^{22}$.

\section{La persistance d'un discours critique sur le placement}

Il semble s'être établi chez nous un véritable rapport dialectique entre, d'une part, les faits, qui révèlent une propension certaine au placement, et le discours, résolument critique à cet égard et ce, depuis plusieurs décennies. 
C'est dans le rapport de la Commission des assurances sociales de Québec, paru en 1933, qu'on trouve énoncées les premières préoccupations en la matière. Les commissaires reprennent les propos de Charlotte Whitton, secrétaire du Canadian Council on Child and Family Welfare, voulant que l'enfant ait droit à une protection qui «... doit lui être donnée par sa famille et dans sa famille ${ }^{23} »$. Ils déplorent l'intervention souvent tardive des autorités publiques dans certaines situations d'abus, recommandent que les associations qui fournissent de l'aide à domicile bénéficient des mêmes privilèges que les institutions ${ }^{24}$ et proposent la mise en place d'un régime d'assistance aux mères nécessiteuses ${ }^{25}$.

Durant la décennie suivante, une autre commission d'enquête (la Commission d'assurance-maladie), dont le rapport paraît en 1944 , se penche sur le problème de la protection de l'enfance. Là encore, les commissaires mettent l'accent sur la préservation de la famille, "milieu logique naturel et approprié où un enfant doit être élevé». Ils dénoncent le recours exagéré au placement institutionnel, "méthode facile et beaucoup trop répandue ${ }^{26}$ ». Ils proposent un avant-projet de loi de la protection de l'enfance prévoyant de nouvelles mesures d'aide aux enfants en difficulté et à leurs familles, notamment le maintien de l'enfant dans son milieu familial sous la surveillance d'une société de protection de l'enfance ${ }^{27}$.

En 1956, dans le cadre d'une Commission royale d'enquête chargée de faire la lumière sur certains problèmes constitutionnels, le père Gonzalve Poulin, premier directeur de l'École de service social de l'Université Laval, rédige une étude exhaustive sur l'assistance sociale dans la province de Québec. Il y affirme que «la dispersion de la famille par le placement institutionnel, quand il n'est pas exigé par des conditions particulières au foyer, répugne à une saine politique familiale qui reconnaît à la famille, même indigente, des responsabilités éducatives vis-à-vis de ses enfants et l'aide financièrement, au besoin, à les remplir ${ }^{28}$ ».

De nombreuses autres études considèrent le placement institutionnel ou en "foyer nourricier» comme une solution de dernier recours à utiliser seulement lorsque les autres ressources sont épuisées ou inappropriées à la situation ${ }^{29}$; plusieurs préconisent le développement de mesures alternatives de soutien susceptibles de favoriser le maintien de l'enfant dans sa famille ${ }^{30}$. 
Ce discours est abondamment repris et confirmé au cours des années 1990, tant par de nombreux groupes d'étude que par l'État lui-même. Il y a d'abord, en 1990, l'avis du Conseil des affaires sociales, dont nous avons déjà fait état en introduction, qui tire la sonnette d'alarme ${ }^{31}$. Puis, en 1990-1991, trois groupes de travail (Harvey, Bouchard et Jasmin), chargés par le gouvernement d'étudier divers aspects de la protection de la jeunesse, insistent sur la nécessité de réduire le recours au placement et de mettre en place des services et des ressources qui permettent, dans toute la mesure du possible, aux enfants de rester dans leur famille et aux parents d'exercer pleinement toutes leurs responsabilités $^{32}$. Les documents publiés en 1995 par le ministère de la Santé et des Services sociaux et d'autres organismes et auxquels nous avons fait référence en introduction abondent dans le même sens; de plus, ils font état de projets-pilotes ayant permis, grâce à la mise en place de programmes appropriés, le maintien dans leur famille d'enfants qui, faute de ces ressources, auraient probablement été placés; finalement, ces documents suggèrent des mesures concrètes susceptibles d'être mises en œuvre à l'échelle du Québec afin d'atteindre cet objectif ${ }^{33}$.

De 1945 à 1994, on constate donc un aller-retour dans les taux de placement d'enfants au Québec. Bien qu'en 1994 ce taux semble être à son plus bas niveau, il se maintient au-dessus de la barre des $6 / 1000$, ce qui est relativement élevé comparativement au taux de 4,4/1000 qu'aurait connu l'Ontario en $1989^{34}$. L'appropriation d'un discours critique à l'égard du placement par un Etat-providence en déclin, qui voit dans la mise en place de programmes de soutien susceptibles de réduire le nombre de placements une façon de comprimer ses dépenses, contribuera sans doute à réduire l'écart observé.

Quoi qu'il en soit, il importe de remonter le cours de l'histoire pour identifier divers facteurs d'ordre social et culturel qui peuvent expliquer l'évolution particulière du Québec au regard du placement d'enfants.

\section{QUELQUES FACTEURS EXPLICATIFS DE LA SITUATION}

Certains traits caractéristiques de nos lois et de nos institutions ont pu contribuer à créer un contexte favorable au placement et à freiner le développement de ressources alternatives. 


\section{Une lente évolution législative en matière de protection de I'enfance et de rapports familiaux}

C'est en 1869 que le législateur québécois intervient pour la première fois dans ce domaine, en adoptant l'Acte concernant les écoles d'industrie ${ }^{35}$. Ce texte législatif organise le placement dans des «écoles industrielles» des enfants "orphelins, errants ou incontrôlables ». Les enfants concernés sont conduits devant un juge qui, après enquête sommaire, peut ordonner un tel placement. Après un séjour d'une durée minimale en institution, l'enfant peut être autorisé à loger chez ses parents ou être placé en apprentissage ou à gages sous la supervision de l'école.

Sous réserve de quelques modifications subséquentes relatives aux catégories d'enfants visés (à la nomenclature initiale s'ajoutent les enfants sévèrement négligés ou maltraités) et aux modalités procédurales et financières de leur placement, cette loi demeurera substantiellement la même jusqu'au milieu du siècle suivant. Elle est centrée sur le retrait de l'enfant de sa famille et sur le placement de celui-ci dans un établissement spécialisé. Il n'est aucunement question de suivi familial ou de mesures de soutien. Déjà, en 1933, la Commission des assurances sociales déplore le fait que cette loi ne prévoit que l'intervention judiciaire et encore seulement dans des cas extrêmes «au point qu'il faut détacher l'enfant de son milieu quand, par une action auprès de ceux qui en sont responsables, on aurait pu empêcher des abus que l'on ne fait que constater ${ }^{36}$ ».

En 1944, à la suite du rapport de la Commission d'assurance-maladie, le gouvernement Godbout fait adopter la Loi de la protection de l'enfance ${ }^{37}$, un texte législatif d'avant-garde pour l'époque, qui prévoit entre autres l'établissement sur le territoire du Québec de sociétés de protection de l'enfance, la création d'une cour familiale et la diversification des mesures susceptibles d'être prises à l'égard des enfants en difficulté et de leur famille. Toutefois, en raison du retour au pouvoir de Maurice Duplessis, quelques mois après son adoption, cette loi ne sera jamais mise en ouvre ${ }^{38}$.

Il faut donc attendre 1950 avant qu'interviennent des changements législatifs notables dans le domaine. On assiste alors à l'adoption de la Loi relative aux écoles de protection de la jeunesse ${ }^{39}$, qui étend la protection de l'État à tout enfant de plus de six ans et de moins de dix-huit ans "particulièrement exposé à des dangers moraux ou physiques, en raison de son milieu 
ou d'autres circonstances spéciales ». Ce texte législatif, qui remplace la Loi des écoles d'industrie et dont l'application est confiée à la nouvelle Cour de bien-être social ${ }^{40}$, demeure toutefois centré sur le placement des enfants concernés dans des écoles spéciales. L'année suivante, il sera modifié de manière à élargir l'éventail des mesures applicables aux enfants de moins de dix-huit ans en besoin de protection.

Le magistrat peut alors, suivant les circonstances et après consultation, s'il y a lieu, auprès d'une agence sociale reconnue par le ministre, laisser l'enfant en liberté surveillée, le confier à toute agence sociale, société, institution, recommander son placement dans une école, ou prendre toute autre décision dans le meilleur intérêt de l'enfant ${ }^{41}$.

Cette modification entraîne du même coup la reconnaissance légale des divers organismes de bienfaisance œuvrant dans le domaine de la protection de l'enfance. En 1960, le titre de la loi est changé pour celui de Loi de la protection de la jeunesse ${ }^{42}$, sans toutefois que son contenu connaisse de modifications importantes.

Ce n'est donc qu'à partir de 1951 que des mesures autres que le placement institutionnel sont prévues par la loi et que des organismes d'assistance polyvalents y sont officiellement reconnus. On peut par ailleurs se demander si les «agences sociales» auxquelles la loi fait référence disposent de ressources suffisantes pour faire de l'action préventive.

La notion de puissance paternelle consacrée par le Code civil du Bas-Canada de 1866 et demeurée pratiquement inchangée jusqu'en 1977 est un autre facteur qui peut expliquer le développement tardif au Québec de services de soutien à domicile. Le Code civil consacre en effet l'autorité absolue du père de famille sur ses enfants. Aucune disposition ne prévoit la déchéance de la puissance paternelle. Les comportements parentaux ne sont réglementés à l'origine que par les lois criminelles. Quant à l'Acte concernant les écoles d'industrie, il s'intéresse surtout aux enfants sans famille ainsi qu'aux enfants "récalcitrants » que même leur propre père peut signaler à l'attention des autorités publiques. Par la suite, cette loi s'appliquera également aux enfants victimes de négligence ou de mauvais traitements graves, mais, comme nous l'avons vu plus haut, l'intervention de l'État se traduira par le placement de l'enfant en institution. Si le pouvoir reconnu officiellement au père de famille peut s'accommoder d'une mesure de ce type, d'ailleurs 
fréquemment sollicitée par la famille elle-même, il ne saurait tolérer une quelconque intrusion de l'État ou de tout autre intervenant dans la vie privée de la famille. La demeure familiale représente un lieu presque sacré, dont seuls quelques rares privilégiés, en l'occurrence les religieux et les membres du clergé, peuvent forcer l'entrée ${ }^{43}$.

Encore aujourd'hui, certains aspects de notre système juridique, notamment l'absence d'une disposition autorisant dans certains cas l'expulsion de la personne abusive - souvent le père ou la mère - du domicile de l'enfant et le caractère imprécis des critères permettant de déclarer un enfant adoptable et, donc, de rompre le lien parental, contribuent à maintenir un taux élevé de placement ${ }^{44}$.

La solidarité familiale que dicte l'obligation alimentaire entre ascendants et descendants limite également les interventions de l'État dans la vie familiale. En obligeant les membres de la famille à subvenir aux besoins essentiels les uns des autres, le Code civil de 1866 confie à celle-ci le rôle premier en matière d'assistance sociale, toute autre institution ne pouvant exercer qu'un rôle supplétif. L'influence déterminante de ces dispositions dans le maintien des structures traditionnelles d'assistance a d'ailleurs été soulignée par diverses commissions d'étude ${ }^{45}$. Pendant longtemps, l'État ne se sentira pas directement concerné par la pauvreté des familles. Il ne leur fournira pas le soutien matériel qui aurait pu justifier qu'il s'immisce dans leur vie quotidienne. Ce rôle sera longtemps dévolu aux autorités religieuses et à leurs institutions. Notons que le nouveau Code civil du Québec (1994) a reconduit l'essentiel de ces dispositions. Très peu appliquées durant la période d'émergence de l'Étatprovidence, celles-ci pourraient retrouver une certaine portée à la faveur de la crise actuelle des finances publiques.

Enfin, bien que la première loi québécoise de l'adoption, adoptée en $1924^{46}$, permette à des couples mariés et même, à certaines conditions (écart d'âge et identité de sexe), à des veufs, veuves ou personnes célibataires d'adopter des enfants, elle comporte des restrictions relatives notamment à l'identité de confession religieuse entre adoptant et adopté. De plus, dans les faits, les sociétés d'adoption recrutent les parents adoptifs en fonction de critères sévères relatifs au statut matrimonial, à la situation matérielle et aux caractéristiques résidentielles. Un grand nombre d'enfants abandonnés ne peuvent donc être adoptés et sont, par conséquent, placés, le plus souvent en institution, dans les 
années 1930 et 1940. De plus, un grand nombre des enfants confiés à des crèches ou à des orphelinats sont «réservés » par leur mère naturelle qui espère les reprendre avec elle un jour ${ }^{47}$.

\section{La place longtemps prépondérante de l'Église catholique dans le champ de l'assistance}

Jusque dans les années 1940, c'est l'Église catholique qui, du moins en milieu canadien-français, assume la presque totalité des responsabilités publiques dans le domaine de l'assistance sociale. Bien que la famille et la paroisse soient les premières à remplir ce rôle ${ }^{48}$, lorsqu'elles sont incapables de faire face à la situation ce sont les communautés religieuses qui prennent la relève, offrant des services de type institutionnel, conformément à leurs traditions ${ }^{49}$. Il existe aussi une certaine forme d'assistance à domicile, destinée aux pauvres, qui se traduit notamment par l'implantation, à partir du XIX ${ }^{\mathrm{e}}$ siècle, de conférences de SaintVincent-de-Paul.

L'urbanisation rapide de la province liée à la révolution industrielle et les problèmes sociaux découlant de la Première Guerre mondiale font toutefois ressortir les faiblesses et les limites de la structure traditionnelle d'assistance ${ }^{50}$. Débordées, aux prises avec de graves difficultés financières, les institutions sont incapables de répondre aux besoins de l'heure. C'est alors que le gouvernement Taschereau fait adopter la Loi de l'assistance publique ${ }^{51}$.

Ce texte législatif répartit également les dépenses des institutions d'assistance publique entre le gouvernement, les municipalités et les institutions elles-mêmes, dont il diminue le fardeau financier d'autant. La loi vise «toute institution qui reçoit, garde, soigne ou hospitalise gratuitement des indigents...». Les services à domicile ne sont cependant admissibles au soutien prévu par la loi qu'en cas d'urgence ou de nécessité absolue et sur recommandation du Service de l'assistance publique. Ce mécanisme a certes favorisé l'institutionnalisation au détriment de services plus diversifiés adaptés à la situation des enfants concernés $^{52}$. La Grande Dépression des années 1930 et la gravité des problèmes sociaux qu'elle engendre amène toutefois divers organismes d'aide à domicile à réclamer un soutien accru de l'État. En 1932, à la suite de pressions exercées par des groupes de bienfaisance du milieu anglophone, l'assistance à domicile obtient une reconnaissance officieuse, les agences sociales étant désormais considérées comme des «institutions sans murs ${ }^{53}$ ». 
Par la suite, des organismes analogues se développent dans le milieu francophone. Montréal, Québec et les autres agglomérations importantes du Québec en voient progressivement l'implantation dans les années 1930 et 1940. Ces ouvres s'intègrent dans le contexte social et religieux de l'époque: elles sont officiellement catholiques, "l'évêque du diocèse y a droit de veto et... rien ne peut $s^{\prime} y$ faire sans son consentement ${ }^{54}{ }$.

Cette nouvelle orientation des services d'assistance n'est pas sans susciter une certaine méfiance dans les milieux cléricaux. Si certains prêtres et religieux acceptent de prendre ce virage et y participent activement, d'autres se montrent très réticents à l'égard notamment de la laïcisation du personnel et des structures de services qu'impliquent les agences nouvellement créées. Le service social «se présentait à l'époque... comme un intrus étranger qui s'opposait à nos traditions, à nos façons de voir et de faire les choses, et mettait en cause l'existence même de nos institutions par les idéologies nouvelles qu'il véhiculait ", écrit à ce propos le père A.-M. Guillemette ${ }^{55}$.

En milieu canadien-français, les agences sociales resteront largement sous la tutelle des autorités religieuses jusqu'à la Révolution tranquille. Dans les faits, elles seront obligées de voir à la distribution et à la supervision des diverses prestations sociales versées par l'État. Cette contrainte, qui ne disparaîtra qu'après 1960 freinera le développement de services de soutien autres que financiers aux familles ${ }^{56}$.

\section{Le développement relativement récent des savoirs et des pratiques dans le domaine de l'aide à I'enfance et à la famille}

Comme nous venons de le voir, le service social se développe plus tardivement en milieu canadien-français qu'en milieu anglophone. Déjà, en 1918, l'Université McGill se dote d'une école de service social ${ }^{57}$. Il faut attendre 1940 et 1943 pour que l'Université de Montréal et l'Université Laval ouvrent de telles écoles ${ }^{58}$.

Les réticences de nombreux clercs à voir des laïcs spécialisés investir ce domaine d'activité peuvent expliquer en partie le développement plus lent dans notre milieu des pratiques professionnelles de service social. Plusieurs aspects de la nouvelle profession sont étrangers, voire en opposition aux valeurs qui sous-tendent l'action de l'Église dans le domaine du bien-être. 
Formation scientifique et rémunération sont deux réalités difficilement conciliables, du moins à première vue, avec charité et bénévolat. Il est en outre vu d'un mauvais œil que religieux et laïcs soient appelés à se côtoyer dans des écoles universitaires où sont susceptibles de s'exprimer divers points de vue ${ }^{59}$.

On se rappelle l'obligation imposée à l'origine aux agences de voir à la distribution de certaines prestations sociales. Même après qu'elles eurent été libérées de ce fardeau, au début des années 1960, leur personnel ne parvient pas à se consacrer autant qu'il le souhaiterait à des fonctions de prévention et de réhabilitation. Des préoccupations professionnelles nombreuses à cette époque où les travailleurs sociaux viennent de se constituer en corporation $^{60}$ et l'absence de lignes directrices clairement définies au sein des agences retardent la réflexion sur l'orientation des services. À ces facteurs s'ajoutent les contraintes budgétaires auxquelles les agences font face et le fait que les quelques travailleurs sociaux qu'on y retrouve occupent principalement des postes administratifs ${ }^{61}$.

À l'aube des années 1970, le bilan des services sociaux québécois demeure mitigé : manque d'accessibilité, inexistence de certains services, absence de politiques claires et cohérentes, pénurie de professionnels, autant de problèmes qui appellent des réponses urgentes. L'adoption, en 1971, de la Loi sur les services de santé et les services sociau ${ }^{62}$ et la prise en charge par l'État de l'ensemble du réseau entraînent une réorganisation complète des services qui accapare toutes les énergies. D'autre part, le nouveau texte de loi reconduit largement le "modèle hospitalier »; l'accent y est mis sur l'hébergement des enfants en difficulté ${ }^{63}$.

Pendant ce temps, «les stratégies d'intervention sociale auprès des jeunes demeurent tout à fait artisanales ${ }^{64}{ }$. Les centres d'accueil de réadaptation font notamment l'objet de vives critiques par le comité Batshaw, qui dépose son rapport en $1975^{65}$. Dans la seconde moitié des années 1970, une nouvelle dynamique se manifeste. Les interventions de réadaptation se structurent et se diversifient pour répondre plus adéquatement aux besoins des jeunes. "À mesure que se consolide l'organisation des services sociaux..., de nouvelles philosophies d'intervention émergent. Dans un courant de désinstitutionnalisation, on remarque que le placement devient un moyen d'aide à l'intervention sociale plutôt qu'un objectif et on assiste à une réduction importante du nombre d'enfants placés... ${ }^{66}$.» 
À cette lente évolution de l'orientation des services correspond un développement tardif de nouvelles méthodes d'intervention auprès des familles. Si, durant toutes ces années, la reconnaissance du principe du maintien de l'enfant dans sa famille trouve écho dans de nombreuses enquêtes et études, la perception des effets négatifs du placement ne se précise, quant à elle, que progressivement. Le développement des sciences sociales et notamment de la psychologie joue sans conteste un rôle majeur à cet égard. Alors qu'en 1933 la commission Montpetit opine qu'il n'est pas "prouvé que l'institution comprime la personnalité et rompe les liens familiaux ${ }^{67}$ ", nombre d'études décrivent par la suite les effets néfastes du placement sur l'enfant et sa famille, l'une d'elles allant jusqu'à affirmer que "pour l'enfant, le traumatisme de son placement risque d'être plus nocif que sa situation de négligence ${ }^{68} »$.

Autre explication au fait que ce discours critique tarde à se traduire dans la réalité, c'est surtout à partir des années 1980 que les intervenants sociaux peuvent recevoir une formation adéquate en "approche familiale», laquelle semble davantage disponible à l'extérieur des centres universitaires ${ }^{69}$. Quoi qu'il en soit, l'élaboration de nouvelles méthodes d'intervention, soutenues par des cadres d'analyse rigoureux, ne peut qu'élargir la gamme des services offerts à la famille et favoriser du même coup le maintien de l'enfant dans son milieu.

\section{CONCLUSION}

Que conclure après ce tour d'horizon de divers aspects de l'évolution du placement des enfants au Québec? Les données disponibles tendent certes à démontrer qu'on a souvent retiré des enfants de leur milieu familial pour les placer en institutions ou, plus tard, en familles d'accueil, alors que, dans bien des cas, une intervention de soutien auprès de l'enfant et de sa famille aurait pu éviter cette mesure extrême. Cette propension au placement s'explique en grande partie par la prédominance du «modèle institutionnel», héritier d'une longue tradition dans le Québec francophone et catholique. La lente évolution des services sociaux et le développement tardif de certaines formes d'intervention auprès des familles ont contribué à la persistance de cette tendance. Tout cela avec, en toile de fond, le caractère sacré de la puissance paternelle et les traits culturels qui en 
découlent. La convergence de tous ces éléments a souvent produit des conséquences paradoxales, puisque l'absence d'intervention préventive auprès des familles a pu, dans bien des cas, provoquer la désintégration de celles-ci. Parlant des débats sociaux qui ont eu cours dans les années 1940, le père Guillemette a pu tenir ces propos éloquents :

Chose remarquable, c'est à ce moment que nous avons pris conscience que le milieu canadien-français, qui se flattait d'une philosophie familiale et d'une tradition familiale exemplaires, était en fait anti-familial par le type de services à l'enfance qu'il imposait à la population $^{70}$.

Il aura fallu la Révolution tranquille, les transformations sociales et culturelles que l'on connaît, les changements législatifs des années 1070, l'émergence de nouvelles approches familiales et, plus près de nous, la crise financière de l'État pour renverser cette tendance.

\section{Notes}

1. Voir, notamment: R. Pauzé, S. Béchard et J. Toupin (1993), «Facteurs de risque associés au placement d'enfants en milieu substitut : recension d'écrits ", $\mathrm{n}^{\circ} 16$, Apprentissage et socialisation, p. 203 et 206 ; J. Boudreau (1993), «A-t-on le choix? Des mesures alternatives: un critère déterminant dans la décision de placement », Prisme, n 3, p. 490 et 497 ; C. Dagenais et C. Bouchard (1993), «Intervention massive ou intervention magique? Les programmes de soutien intensif aux familles », Prisme, n ${ }^{\circ} 3$, p. 503.

2. Conseil des affaires sociales (1990), De la protection des enfants au soutien des parents, Québec, p. 11 et 14 . Il est à noter que le taux de 8,4/1000 enfants placés au Québec ne tient pas compte des placements effectués en vertu de la Loi sur les jeunes contrevenants; par ailleurs, le taux ontarien de 4,4/1000 enfants placés ne concerne, lorsqu'il s'agit de placements de protection, que les enfants de moins de seize ans.

3. Commission des assurances sociales de Québec (1933), 2e rapport, Québec, p. 4 et 13 notamment.

4. Ministère de la Santé et des Services sociaux (1995), Maintenant et pour l'avenir... La jeunesse, Québec, ch. 4 ; Vers un continuum de services intégrés à la jeunesse (1995), Québec (en particulier le rapport du comité provincial sur les projets expérimentaux de solutions de rechange au placement des jeunes).

5. Ces données résultent de la mise en rapport du nombre d'enfants placés et de la population totale des jeunes de moins de dix-huit ans pour les années considérées. Elles ne tiennent pas compte des placements informels effectués, par exemple, dans la famille élargie, le voisinage ou la communauté paroissiale. Nos sources sont: pour 1945 l'Annuaire statistique du Québec, 1945-1946 et 1953 et l'ouvrage de C.-É. Bourgeois, Une richesse à sauver: l'enfant sans soutien, 
Trois-Rivières, Éditions du bien public, 1947 ; pour 1960 et 1965, le Recensement du Canada, 1961 et 1966 et une étude publiée par le Centre de services sociaux Laurentides-Lanaudière, L'expérience du placement d'adolescents et d'adolescentes en famille d'accueil, Sainte-Thérèse, 1980 ; pour 1972, le Recensement du Canada, 1971, et un document publié par le Ministère des Affaires sociales (Comité de la santé mentale), Avis sur les enfants placés, Québec, 1981 ; pour 1978, les Estimations intercensitaires, 1976-1981 de Statistique Canada et l'Avis précédemment décrit; à partir de 1984, le nombre d'enfants placés est tiré des documents annuels produits par les Centres de services sociaux (maintenant les Centres jeunesse) intitulés État de la situation au 31 mars... et le nombre total d'enfants de moins de dix-huit ans provient de divers documents publiés par Statistique Canada : Estimations intercensitaires, 1981-1986, Estimations annuelles postcensitaires, $1^{\text {er }}$ juin 1989 et $1^{\text {er }}$ juin 1991, Statistiques démographiques annuelles, 1993 et 1994.

6. Commission des assurances sociales de Québec, $2^{\mathrm{e}}$ rapport, p. 34.

7. A. Saint-Pierre, L'Euvre des Congrégations religieuses de charité dans la province de Québec, Montréal, Bibliothèque canadienne, 1930, p. 22, cité par C.-E. Bourgeois, Une richesse..., op. cit., p. 107.

8. Sour Sainte-Rita-de-Cascia (1951), Adaptation sociale de l'orpheline (extraits d'une thèse de maîtrise présentée en 1951 à l'École de service social de l'Université Laval), Service social, vol. 3, p. 137.

9. M. Lachapelle, Facteurs qui ont entouré les placements d'enfants hors du milieu familial considérés par la sauvegarde de l'enfance (thèse de maîtrise présentée en 1952 à l'École de service social de l'Université Laval), cité par J.-L. Roy (1976), La marche des Québécois : le temps des ruptures, 1945-1960, Montréal, Leméac, p. 46.

10. Conseil des CEuvres de Montréal (1964), 7000 enfants placés hors de leur foyer: étude évaluative des placements d'enfants de langue française de l'île de Montréal en janvier 1963, Montréal, Service de recherche du Conseil des Euvres de Montréal, p. 51.

11. Ministère des Affaires sociales (1979), Opération 30 000, Québec, p. 280 et s.

12. R. Pauzé, S. Béchard et J. Toupin, «Facteurs de risque...», op. cit., p. 205.

13. C.-E. Bourgeois, Une richesse..., op. cit, p. 112 et 113.

14. M. Lachapelle, dans J.-L. Roy, La marches des..., op. cit., p. 46.

15. Conseil des Euvres de Montréal, 7000 enfants..., op. cit., p. 51 ; Ministère des Affaires sociales, Opération 30 000, op. cit., p. 327-328.

16. R. Pauzé, S. Béchard et J. Toupin, «Facteurs de risque...», op. cit., p. 205.

17. M. Lachapelle, dans J.-L. Roy, La marche des..., op. cit., p. 46.

18. Conseil des CEuvres et du Bien-être de Québec, Étude des foyers nourriciers et de leur population dans le diocèse de Québec (1968), Québec, Service de recherche du Conseil des Euvres p. 60-62.

19. Statistique Canada, Recensement du Canada, 1961 et 1971.

20. Centre de services sociaux Laurentides-Lanaudière, L'expérience de placement..., op. cit., p. 4.

21. Jean-Guy Boutin, «Étude comparative des statistiques de placements d'enfants au Québec et en Ontario », Administration hospitalière et sociale, octobre 1974, p. 7-11.

22. Centre de services sociaux Laurentides-Lanaudière, L'expérience de placement..., op. cit., p. 5. 
23. Commission des assurances sociales de Québec, $2^{\text {e }}$ rapport, p. 4 et 13.

24. La Loi de l'assistance publique que le gouvernement Taschereau a fait adopter en 1921 ne vise que les institutions. Les organismes de soutien à domicile ne peuvent s'en prévaloir qu'exceptionnellement et en situation d'urgence.

25. Le premier gouvernement Duplessis fait adopter, en 1937, la Loi de l'assistance aux mères nécessiteuses. Les critères retenus à l'origine pour l'admissibilité aux prestations sont restrictifs et moralisateurs. La nouvelle loi ne produit donc pas tous les effets escomptés : trop d'enfants sont encore placés en raison des difficultés économiques de leur mère.

26. Commission d'assurance-maladie de Québec (1944), $1^{\text {er }}$ rapport, Québec, p. 8.

27. Cette recommandation est suivie par l'adoption d'une loi qui ne sera toutefois jamais appliquée. Voir plus bas, notes 34 et 35 .

28. G. Poulin (1955), L'assistance sociale dans la province de Québec 1608-1951, Commission royale d'enquête sur les problèmes constitutionnels, Annexe 2, Québec, p. 90.

29. Notamment R. Rivard et G. Lacroix (1958), Essai de normes professionnelles des foyers nourriciers, Commission «Enfance Caritas-Canada», Montréal; Conseil des Euvres de Montréal, 7000 enfants placés..., op. cit.

30. Notamment Conseil des Euvres et du Bien-être de Québec, Étude des foyers nourriciers..., op. cit.; Gouvernement du Québec (1972), Rapport de la Commission d'enquête sur la santé et le bien-être social, vol. 6, t. 1, Québec; Ministère des Affaires sociales (1975), Rapport du Comité d'étude sur la réadaptation des enfants et des adolescents placés en centre d'accueil, Québec.

31. Conseil des affaires sociales, De la protection des enfants..., op. cit.

32. Gouvernement du Québec, ministère de la Santé et des Services sociaux, $L a$ protection sur mesure, un projet collectif (rapport Harvey II), p. 114-115; en annexe à ce rapport, on retrouve par ailleurs un texte émanant du même ministère et intitulé «Protocole relatif aux activités entourant le placement d'un enfant ", lequel propose des balises destinées à limiter le placement des enfants et à réserver cette mesure aux seules situations pour lesquelles n'existe aucune solution alternative valable ; Gouvernement du Québec, ministère de la Santé et des Services sociaux (1991), Un Québec fou de ses enfants, Rapport du groupe de travail pour les jeunes (rapport Bouchard), Québec, p. 168 et 170 ; Gouvernement du Québec, ministère de la Santé et des Services sociaux et ministère de la Justice (1992), La protection de la jeunesse, plus qu'une loi, Rapport du groupe de travail sur l'évaluation de la loi sur la protection de la jeunesse (rapport Jasmin) Québec, p. 33 et 78.

33. Ministère de la Santé et des Services sociaux, Maintenant et pour l'avenir... et Vers un continuum de services..., op. cit.

34. Voir données citées en introduction et à la note 1.

35. Acte concernant les écoles d'industrie, S.Q. 1869, ch. 17.

36. Commission des assurances sociales de Québec, $2^{\mathrm{e}}$ rapport, p. 18.

37. Loi concernant la protection de l'enfance, S.Q. 1994, ch. 33.

38. Voir, à ce sujet, Renée Joyal et Carole Chatillon (1994), «La Loi québécoise de protection de l'enfance de 1944 ; genèse et avortement d'une réforme », Histoire sociale / Social History, $\mathrm{n}^{\circ} 53$, p. 33.

39. Loi relative aux écoles de protection de la jeunesse, S.Q. 1950, ch. 11.

40. Loi instituant la Cour de bien-être social, S.Q. 1950, ch. 10.

41. Loi modifiant la Loi des écoles de protection de la jeunesse, S.Q. 1950-1951, ch. 56. 
42. Loi de la protection de la jeunesse, S.Q. 1959-1960, ch. 42.

43. Cette culture familiale «s'accommodait des conseils et des pressions des autorités religieuses », mais ne pouvait "tolérer aussi facilement ceux des intervenants laïcs, mandatés par la justice et les services sociaux»: Alice Parizeau (1979), Protection de l'enfant: échec?, Montréal, Presses de l'Université de Montréal, p. 17.

44. R. Pauzé, S. Béchard et J. Toupin (1993), «Facteurs de risque...», op. cit., p. 208 ; Claude Bilodeau, «Ne tirez pas sur le placeur!», Prisme, n 3, p. 486.

45. Voir, à ce sujet, G. Poulin (1963), L'assistance sociale..., op. cit., p. 40 et Gouvernement du Québec, Rapport du Comité d'étude sur l'Assistance publique, Québec, p. 30.

46. Loi concernant l'adoption, S.Q. 1924, ch. 75.

47. M.-P. Malouin (dir.) (1996), L'Univers des enfants en difficulté au Québec entre 1940 et 1960, Montréal, Bellarmin, p. 388 et 409.

48. «Chaque famille prenait soin de ses infirmes, de ses vieillards et des orphelins de la parenté immédiate, voire du voisinage; chaque paroisse s'occupait de ses pauvres » : Esdras Minville (1950), «L'aspect économique du problème national canadien-français », Montréal, Les Éditions Bellarmin, n 436, p. 6.

49. Des débuts de la colonie jusqu'au milieu du XIX ${ }^{\mathrm{e}}$ siècle, l'hôpital général constitue la principale, sinon l'unique forme d'assistance sociale au Québec; il répond à tous les besoins qui ne peuvent être satisfaits dans le cadre de la famille ou de la paroisse. Au cours du XIX $\mathrm{X}^{\mathrm{e}}$ siècle, les institutions se diversifient (crèches, orphelinats, hospices, école d'industrie), mais sont toujours dirigées par des religieux ou des clercs: G. Poulin (1949), «Évolution des services sociaux de la province de Québec », Bien-être social canadien, $\mathrm{n}^{\circ} 1$, p. 2.

50. Noël Simard (1956), «Répercussions de l'Assistance publique sur la structure et les fonctions des institutions et des œuvres », Bien-être social canadien, vol. 8, p. 37.

51. Loi de l'assistance publique, S.Q. 1921, ch. 79.

52. Pierre Foucault (1993), «Héberger, corriger, réadapter... Une histoire des mesures de protection pour les jeunes au Québec », Prisme, n 3, p. 468-469.

53. «... n'ayant pas les communautés religieuses sur lesquelles s'appuyer, la population non catholique de la Province avait... surtout organisé selon la formule des Charity Organization Societies des agences sociales employant un personnel laïque formé, et régies par des conseils d'administration bénévoles » : Hayda Denault, «L'insertion du service social dans le milieu canadien-français », (1961-1962) Service social, vol. 10, $\mathrm{n}^{\circ} 3$; vol. 11, nº 2, p. 13.

54. Serge Mongeau (1967), Évolution de l'assistance au Québec. Une étude historique des diverses modalités d'assistance au Québec des origines de la colonie à nos jours, Montréal, Éditions du Jour, p. 59.

55. A.-M. Guillemette (1973), «Vingt-cinq ans de service social», Communauté chrétienne, vol. XII, p. 275.

56. G. Lacroix (1966), «Services sociaux à la famille au Québec», Relations, mai, p. 137 ; G. Poulin (1964), "La socialisation des structures de bien-être, phénomène historique ", Culture, décembre, p. 338.

57. S. Mongeau, Évolution de l'assistance..., op. cit., p. 39.

58. N. Vanier, "Aperçu historique de l'École de service social de l'Université de Montréal », (1961-1962) Service social, vol. 10, $\mathrm{n}^{\circ} 3$; vol. 11, $\mathrm{n}^{\circ} 1$, p. 95-101; H. Denault, "L'insertion du service social... », op. cit., p. 13.

59. A.-M. Guillemette, «Vingt-cinq ans...», op. cit., p. 276-278. 
60. G. Renaud (1978), L'éclatement de la profession en service social, Montréal, Les Éditions coopératives Albert St-Martin, p. 34-35.

61. Gouvernement du Québec, Rapport de la Commission d'enquête sur la santé et le bien-être social, vol. VI, t. 1, p. 65-68; Fédération des services sociaux à la famille, divers mémoires parus dans La famille, 1964, vol. 1, n 10, p. 30; 1965, vol. 3, no 27 , p. 18 ; 1966, vol. 4, no 38, p. 27, cité dans G. Renaud, L'éclatement..., p. 84.

62. Loi sur les services de santé et les services sociaux, S.Q. 1971, ch. 48.

63. P. Foucault, «Héberger, corriger...», op. cit., p. 470.

64. Y. Vaillancourt, D. Bourque, F. David et E. Ouellet (1987), La privatisation des services sociaux (rapport de recherche présenté à la Commission d'enquête sur les services de santé et les services sociaux par l'INRS), Québec, p. 54.

65. Ministère des Affaires sociales (1975), Rapport du Comité d'étude sur la réadaptation des enfants et des adolescents placés en centre d'accueil, Québec.

66. Gouvernement du Québec (1988), Rapport de la Commission d'enquête sur les services de santé et les services sociaux, Québec, Publications du Québec, p. 231.

67. Commission des assurances sociales de Québec, $2^{\mathrm{e}}$ rapport, p. 46.

68. Ministère des Affaires sociales (Comité de la santé mentale) (1981), Avis sur les enfants placés, Québec; J. Boudreau, «A-t-on le choix?», op. cit., p. 490.

69. Christian Côté (1991), «La thérapie familiale : évolution et enjeux», Intervention $\mathrm{n}^{\circ} 89$, p. 5 et 8 .

70. A.-M. Guillemette, «Vingt-cinq ans...», op. cit., p. 277. 